\title{
Genetic Screening in Pheochromocytoma/Paraganglioma
}

\author{
Peihua Liu ${ }^{1} \cdot$ Xiongbing $\mathrm{Zu}^{1} \cdot$ Longfei Liu $^{1}$
}

Received: 31 March 2016 / Accepted: 16 September 2016/Published online: 3 October 2016

(C) Arányi Lajos Foundation 2016

Dear Editor,

We have read the article "Novel SDHB and TMEM127 Mutations in Patients with Pheochromocytoma/Paraganglioma Syndrome" with great interest [1]. In this article genes screening of SDHB, SDHC, SDHD, SDHAF2, MAX and TMEM127 was performed in Hungarian patients with apparently sporadic PHEO/PGLs using the first generation sequencing technologySanger chain termination method by the authors. With the combination of 4 RET mutations and 4 VHL mutations, a conclusion that the carrier rate of susceptibility genes in Hungarian patients is $21.1 \%$ was made.

However, we suggest that it must be deliberated carefully despite its similarity to previous ones [2]. First of all, not all the susceptibility genes were included in their screening strategy. There are as many as 21 genes from the latest research but genes NF1, SDHA, HIF2A, KIF1B $\beta$, EGLN1, EGLN2, H-RAS, KRAS, IDH1, FH, BAP1, MDH2 and ATRX were excluded [3]. Hence, genes of 12 malignant/recurrent patients and 3 bilateral/ multiple-location patients mentioned as no genetic cause ones in the article may be mutated within above. Secondly, because the research subjects were from single center as well as limited in quantity, it's unable to represent the general mutation of PHEO/ PGLs in the whole Hungarian population. In addition, for the new discoveries of SDHB genotype-phenotype associations pointed out in the article, other authors have summarized the clinical presentations of SDHB-mutated patients before [4].

Longfei Liu

longfei_liu@163.com

1 Department of Urology, Xiangya hospital, Central South University, Changsha, Hunan 410008, China
As far as we are concerned, only under the premise of a larger number of patients and taking whole-exome sequencing technology as a prerequisite for studying PHEO/PGL can we find more pathogenic genes, summarizes more genotypephenotype associations and provide a solider basis for precision medicine in the future.

It's still a difficult and hot spot for the exploration of PHEO/ PGL susceptibility genes, and differential expression of susceptibility genes in different races and ethnicities has not been well studied. This article partly shows the mutation spectrum and mutation frequency of PHEO/PGL susceptibility genes in Hungarian population and is going to encourage more researchers to study PHEO/PGL susceptibility genes of certain race and ethnicity, which will give us a better understanding of PHEO/PGLs in all humanity.

Acknowledgments This study was supported by the National Natural Science Foundation of China (No.81400773) and Natural Science Foundation of China Hunan Province (No.14JJ4024).

Conflicts of Interest The authors declare no conflicts of interest.

\section{References}

1. Patocs A, Lendvai NK, Butz H, Liko I, Sapi Z, Szucs N et al (2016) Novel SDHB and TMEM127 mutations in patients with pheochromocytoma/Paraganglioma syndrome. Pathol Oncol Res 22(4):673-679

2. Kolackov K, Tupikowski K, Bednarek-Tupikowska G (2012) Genetic aspects of pheochromocytoma. Adv Clin Exp Med 21(6): 821-829official organ Wroclaw Medical University

3. Jochmanova I, Zhuang Z, Pacak K (2015) Pheochromocytoma: gasping for air. Horm Cancer 6(5-6):191-205

4. Santos P, Pimenta T, Taveira-Gomes A (2014) Hereditary Pheochromocytoma. Int J Surg Pathol 22(5):393-400 